\title{
BMJ Open Haemorrhage control in the prehospital setting: a scoping review protocol
}

To cite: Ezeibe C, McCarty JC, Chaudhary MA, et al. Haemorrhage control in the prehospital setting: a scoping review protocol. BMJ Open 2019;9:e029051. doi:10.1136/ bmjopen-2019-029051

- Prepublication history and additional material for this paper are available online. To view these files, please visit the journal online (http://dx.doi org/10.1136/bmjopen-2019029051).

Received 10 January 2019 Revised 5 April 2019 Accepted 5 June 2019

Check for updates

(C) Author(s) (or their employer(s)) 2019. Re-use permitted under CC BY-NC. No commercial re-use. See rights and permissions. Published by BMJ.

${ }^{1}$ Center for Surgery and Public Health, Brigham and Women's Hospital, Harvard Medical

School, Boston, Massachusetts, USA

${ }^{2} \mathrm{TH}$ Chan School of Public Health, Harvard University, Boston, Massachusetts, USA

${ }^{3}$ James Cook University College of Medicine and Dentistry,

Townsville, Queensland,

Australia

${ }^{4}$ Department of Emergency

Medicine, Brigham and

Women's Hospital, Harvard

Medical School, Boston,

Massachusetts, USA

Correspondence to

Dr Eric Goralnick;

egoralnick@bwh.harvard.edu

\author{
Chibuike Ezeibe, ${ }^{1}$ Justin C McCarty, ${ }^{1,2}$ Muhammad A Chaudhary, ${ }^{1}$ \\ Elzerie De Jager, ${ }^{1,3}$ Juan Herrera-Escobar, ${ }^{1,2}$ Tomas Andriotti, ${ }^{1,2}$ Molly P Jarman, ${ }^{\oplus 1}$ \\ Gezzer Ortega, ${ }^{1}$ Eric Goralnick ${ }^{1,4}$
}

\begin{abstract}
Introduction Prehospital haemorrhage control has saved thousands of lives in the military over the last decade. While uncontrolled haemorrhage is a leading cause of preventable injury death in the USA for individuals under 45 , military prehospital haemorrhage control techniques have not fully translated to the civilian sector in the USA. The effective implementation of haemorrhage control for civilian prehospital trauma is dependent on a more complex array of system and personnel-level factors than the military.

Objective This protocol describes the methodology of a scoping review on haemorrhage control strategies in the prehospital setting; specifically, education, logistics and implementation of these strategies. The aim of the review is to identify research gaps and create recommendations for future research surrounding prehospital layperson haemorrhage control.
\end{abstract}

Methods The protocol uses the framework published by The Joanna Briggs Institute and Arksey and O'Malley, while following the Preferred Reporting Items for Systematic Review and Meta-Analysis Extension for Scoping Review Protocols guidelines. The search strategy was refined with the help of a medical librarian. Three peer-reviewed databases (EMBASE, PubMed and Web of Science), databases dedicated to grey literature sources, and reference mining will be used. Two investigators will independently screen and extract data. Discrepancies will be resolved by a third investigator. The extracted data will undergo descriptive analysis of the contextual data and a quantitative analysis using the appropriate statistical methods. In addition, this search strategy will be supplemented by a grey literature search.

Ethics and dissemination Research ethics approval is not required for this scoping review. This scoping review will serve to highlight existing gaps within the literature to guide further research and develop future strategies to improve prehospital haemorrhage management. The results of this review will be presented at relevant national and international conferences and published in a peerreviewed journal.

\section{INTRODUCTION}

In the USA alone, more individuals die from trauma-related incidents (ie, motor vehicle collisions, homicides) compared with any other cause, outside of cardiovascular disease and cancer. ${ }^{2}$ In 2014, there

\section{Strengths and limitations of this study}

The use of peer-reviewed literature, as well as the inclusion of grey literature allows for a wide overview of various study designs and methodologies and highlights the state of existing literature surrounding prehospital bleeding control.

- The use of a scoping review is an effective method for exploring and mapping broad and diverse topics.

- Broad nature of this study type creates difficulty in establishing boundaries with scope.

- Interpreting evidence may be challenging with lack of quality appraisal.

were approximately 200000 injury deaths in the USA. Within the cohort of people 1 to 44 years old, traumatic injuries are the most common cause of death and among all ages, injury is the fourth overall leading cause of death. ${ }^{3-5}$ Globally, injuries and violence account for $9 \%$ of the world's deaths, which is approximately twice the number of deaths resulting from HIV/AIDS, tuberculosis and malaria combined. ${ }^{6}$

With trauma-related deaths on the rise following the 2012 Sandy Hook active shooter disaster, the American College of Surgeons convened a committee purposed to create a national protocol for enhanced survivability from active shootings and intentional mass casualty events. The committee's recommendations were aptly named the Hartford Consensus. ${ }^{7}$ Due to these recommendations, secondary prevention (the reduction of the impact of an injury that has already occurred) of trauma death has increasingly become the focus in trauma-related research. ${ }^{4}$ The impact of secondary prevention on external haemorrhage was recognised as a major cause of potentially preventable death following injury. The lifesaving benefits of prehospital haemorrhage control are illustrated by the US Military Tactical Combat Casualty Care (TCCC) Committee's efforts to reduce mortality from external haemorrhage during 
the conflicts in Iraq and Afghanistan. ${ }^{8}$ These responses include the development of new haemostatic agents like chitosan-based haemostatic gauze dressings and the policy recommendations that all deploying combatants and military medical personnel receive TCCC training and carry extremity tourniquets. ${ }^{9} 10$

The integration of these guidelines into military practices has been associated with decreased preventable deaths resulting from limb external haemorrhage. One such study that attests this claim is an analysis of battle injury data collected during combat deployments to examine casualties from the 75th Ranger Regiment, US Army Special Operations Command during conflicts in Afghanistan and Iraq from October 2001 through March $2010 .{ }^{11}$ According to Kotwal et al, among all personnel who were trained in TCCC within the regiment, a total of 419 battle injury casualties were incurred during the years of continuous combat, with the regiment's rates of $10.7 \%$ killed in action and $1.7 \%$ who died of wounds which were lower than the department of Defense rates of $16.4 \%$ and $5.8 \%$, respectively. A similar study by Eastridge $e t$ al examined combat casualties in both Iraq and Afghanistan from October 2001 to June 2011. This study examined over 4500 battlefield fatalities and identified $24 \%$ of these fatalities to be potentially survivable (PS), with the analysis associating $90 \%$ of the PS deaths with haemorrhage. ${ }^{12}$ The effectiveness of the policies within military medicine have resulted in a push to implement similar strategies in prehospital haemorrhage control within the civilian sector.

Nationally, uncontrolled bleeding is the leading cause of preventable death in trauma. ${ }^{13}$ The 'Stop the Bleed' campaign, an initiative convened by the National Security Council staff in response to advances made by military medicine and research in haemorrhage control during recent foreign conflict, aims to train and empower laypersons to act as immediate responders to stop bleeding in trauma. ${ }^{14}$ However, while haemorrhage control training has proven its effectiveness within the military, there is insufficient research on the effectiveness of these same techniques within the civilian sector, specifically in the prehospital setting before victims are transported to medical facilities. We consider that, along with a lack of research, there are many other determinants related to the implementation of effective haemorrhage control methods in the prehospital setting.

In this context, our aim is to conduct a scoping review to examine the existing literature pertaining to education, logistics and implementation of prehospital haemorrhage control strategies among various populations and locations, and identify the research gaps that are associated with the literature, as well as develop recommendations for future research surrounding haemorrhage control in prehospital setting.

\section{METHODS}

A scoping review will be conducted to identify and examine the existing research focused on prehospital haemorrhage control. In contrast to systematic literature reviews that aim to answer specific questions, scoping reviews produce a broad overview of the field. As such, our study will be conducted using methodology published by Arksey and O'Malley, as well as the updated framework by The Joanna Briggs Institute. ${ }^{1516}$ As recommended by Tricco $e t$ al, this protocol will follow the relevant aspects of the Preferred Reporting Items for Systematic Review and Meta-Analysis Extension for Scoping Review Protocols (PRISMA-ScR) guidelines to ensure rigour and reduce bias in reporting the methodology. ${ }^{17}$ Using this established protocol, we plan on systematically reviewing the existing literature discussing haemorrhage control in the prehospital setting and mapping key concepts and identifying the need for further research in particular areas within the field. The framework consists of five consecutive stages: (1) identifying the research question, (2) identifying relevant studies, (3) study selection, (4) charting the data and (5) collating, summarising and reporting results.

\section{Stage 1: identifying the research question}

The objective of this review is to identify what gaps exist within the research of prehospital haemorrhage control and identify what recommendations can be made to improve such gaps. Moreover, this study seeks to establish an understanding of how prehospital haemorrhage control interventions are taught and implemented and what factors are key in their implementation for success in settings outside of military medicine. We aim to provide answers for the following sub-questions:

1 . What materials are being used effectively for haemorrhage control in prehospital settings?

2. What issues are there regarding the efficacy and efficient distribution of haemorrhage control materials and devices?

3. What education modalities are being used to teach haemorrhage control?

4. Does successful implementation of haemorrhage control skills differ among geographical locations?

\section{Stage 2: identifying relevant studies}

The search strategy was developed by the research team with a medical librarian. Our literature search was open, including both grey literature (evidence not published in peer-reviewed publications) and peer-reviewed literature. Using the peer-reviewed databases Embase, PubMed and Web of Science, a systematic search for relevant studies was conducted. The search was limited to articles published between the first available record in 1998 until November 2018, given that we wanted to examine prehospital bleeding control interventions over the past 20 years. A non-systematic search (or grey literature search) using reports by government agencies (ie, Canadian Agency for Drugs and Technologies in Health), by search engines designed to find evidence-based content (ie, Turning Research into Practice) or of database purposed for production reports in health services research and 
selected urban health topics (ie, Grey Literature Report) was carried out.

The primary search terms were focused on using variations of the following Medical Subject Heading (MeSH) terms: Emergency Medical Services, Bleeding, Haemorrhage, Shock (Haemorrhagic), Exsanguination, and Prevention \& control. We also used the term 'Prehospital' as a keyword in searches that do not list it as a $\mathrm{MeSH}$ term. These MeSH terms and keywords, along with the addition of relevant subheadings, were systematically combined using Boolean operators (OR, AND, NOT) to capture relevant fields. A complete list of the search terms is presented in online appendix A. The results from the search were exported from each database into referencing software (Mendeley) to remove duplicates. ${ }^{18}$ The remaining papers will be exported to Covidence, a web-based software platform that streamlines the inclusion eligibility screening of systematic reviews. ${ }^{19}$

\section{Stage 3: study selection}

Using Covidence, the investigators will conduct a screening process to determine the eligibility of articles generated during the initial search. This screening process will consist of three rounds of screening: title, abstract and full-text review. The title and abstract screening will be distributed among three pairs of investigators (CE and EDJ, JM and MC, JHE and TA). Discrepancies will be resolved by a third investigator (MJ). Full text screening will be conducted by three investigators (CE, JM and EDJ). This process will be documented as a PRISMA flowchart (figure 1). Additional articles will be identified through reference mining of included studies. Subsequent discussion will follow to establish a consensus of which papers will be included.

The inclusion criteria are:

1. Studies focused on bleeding control interventions performed in the prehospital setting.

2. Commentaries, editorials, letters and studies pertaining to suggestions of interventions that can be implemented.

The key concept is the application of strategies surrounding the management of haemorrhage control outside of the hospital setting, and studies will be considered that evaluate and compare the differences seen in intervention strategies and teaching modalities among various laypersons in multiple settings. Additionally, we will examine the efficiency in managing bleeding with proper application of tools and the skills taught.

Publications written in languages other than English will be excluded. We will also exclude any studies discussing bleeding control management outside of the prehospital setting (in-hospital or perioperative) or specifically examining patients who suffer from pre-existing bleeding disorders and/or are taking anticoagulants for pre-existing conditions.

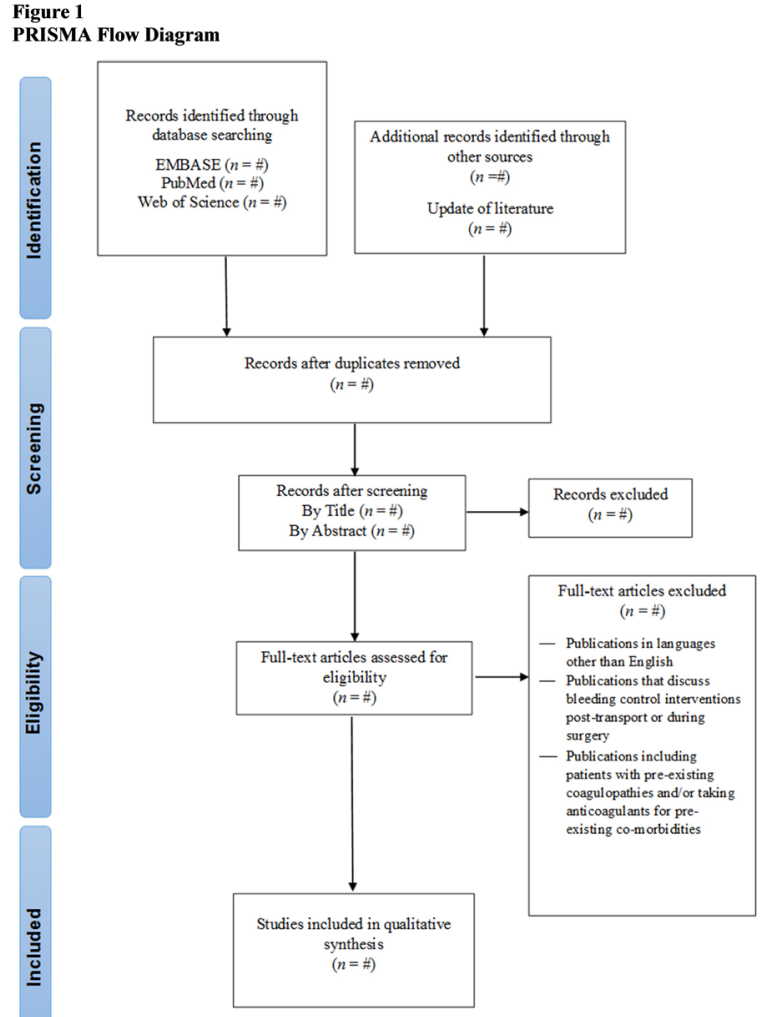

Figure 1 Preferred Reporting Items for Systematic Review and Meta-Analysis flow diagram.

\section{Stage 4: charting the data}

Articles and studies that meet the inclusion criteria will be charted into an Excel spreadsheet for data extraction and collation (table 1). Two reviewers (CE and JM) will input the data as shown below:

If further information is needed, we will contact the authors of the included studies. Any disagreement concerning included studies will be solved by discussion within the research team.

\begin{tabular}{|c|c|}
\hline \multirow[t]{5}{*}{ General information } & Title \\
\hline & Author \\
\hline & Year of publication \\
\hline & Country of origin \\
\hline & Study design \\
\hline Aims/Purpose & Extracted data \\
\hline Methodology & Extracted data \\
\hline $\begin{array}{l}\text { Intervention type, } \\
\text { comparisons and details }\end{array}$ & Extracted data \\
\hline Outcomes/Measures & Extracted data \\
\hline Key findings/Conclusions & Extracted data \\
\hline
\end{tabular}




\section{Stage 5: data summary and synthesis of results}

The general characteristics of each study will be summarised. Studies will be classified according to their target population and the research stage at which they were performed or suggested. The collected findings will provide an overview of the quantity of research rather than an assessment of the quality of individual studies. Moreover, with the studies that are included in our review, we will categorise them into the following: (1) materials/ logistics (2) intervention quality, (3) educational factors, (4) domestic versus global implementation. With these categories, we will identify potential research gaps within the literature and determine which areas require more in-depth analysis.

\section{Patient and public involvement}

This study did not involve patients, apart from those mentioned in the studies identified through our searches. Whereas our study is meant to inform experts and stakeholders of the existing state of science concerning our topic, this data has not been disseminated to the public yet. Following the successful publishing of this protocol, we intend to construct and submit a systematic scoping review to identify gaps existing within the research of prehospital haemorrhage control and identify what recommendations can be made to improve such gaps.

\section{DISSEMINATION AND ETHICS}

As a scoping review, the purpose of our study is to characterise the quantity and quality of existing literature and present an overview of prehospital haemorrhage control rather than a meta-synthesis. Thus, our aim is to examine existing literature surrounding the topic of prehospital haemorrhage control and highlight gaps within the research.

This scoping review is part of a larger project to elucidate gaps in implementation, education and logistics of training, and empowering laypersons to perform bleeding control. The results of this review will serve as a key component for National Stop the Bleed Research Consenus Conference taking place at Brigham and Women's Hospital/Harvard Medical School in February 2019. The objective of this conference is to create a consensus research agenda for use by clinicians, researchers, funding organisations, policy makers and other key stakeholders for years to come. Furthermore, we believe this review could be a critical step in the uniform implementation of haemorrhage control in prehospital trauma management.

Acknowledgements We would like to acknowledge Meredith Solomon, MLS, Harvard Medical School Countway Library for her guidance on scoping review methodology.

Contributors All authors have made substantive intellectual contributions to the development of this protocol. EG conceptualised the review approach and provided general guidance to the research team. All authors were involved in developing the review questions and the review design. CE identified the framework from which JCM and EDJ developed and tested search terms. MAC, JHE and TA initially developed the data extraction framework which was then further developed by input from team members. CE, GO and MPJ initiated the first draft of the manuscript which was then followed by numerous iterations with substantial input and appraisal from all the authors. All authors approve the final version of the manuscript.

Funding This work was supported by the Gillian Reny Stepping Strong Center for Trauma Innovation.

Competing interests None declared.

Patient consent for publication Not required.

Provenance and peer review Not commissioned; externally peer-eviewed.

Open access This is an open access article distributed in accordance with the Creative Commons Attribution Non Commercial (CC BY-NC 4.0) license, which permits others to distribute, remix, adapt, build upon this work non-commercially, and license their derivative works on different terms, provided the original work is properly cited, appropriate credit is given, any changes made indicated, and the use is non-commercial. See: http://creativecommons.org/licenses/by-nc/4.0/.

\section{REFERENCES}

1. Heron MP. Deaths: Leading causes for 2016. 2018.

2. National Center for Injury Prevention and Control. Key Data and Statistics|WISQARS|Injury Center. https://www.cdc.gov/injury/ wisqars/overview/key_data.html

3. Kochanek KD, Murphy SL, Xu J, et al. Deaths: Final Data for 2014. Natl Vital Stat Rep 2016;65:1-122.

4. Davis JS, Satahoo SS, Butler FK, et al. An analysis of prehospital deaths: Who can we save? J Trauma Acute Care Surg 2014;77:213-8.

5. Rhee P, Joseph B, Pandit $\mathrm{V}$, et al. Increasing trauma deaths in the United States. Ann Surg 2014;260:13-21.

6. Organization WH. Injuries and violence: the facts 2014. Geneva: Switz World Heal Organ, 2014.

7. Bleeding Control. The Hartford Consensus. https://www. bleedingcontrol.org/about-bc/hartford-consensus (Cited 18 Dec 2018).

8. Bulger EM, Snyder D, Schoelles K, et al. An evidence-based prehospital guideline for external hemorrhage control: American College of Surgeons Committee on Trauma. Prehosp Emerg Care 2014:18:163-73.

9. Bennett BL, Littlejohn LF, Kheirabadi BS, et al. Management of External Hemorrhage in Tactical Combat Casualty Care: ChitosanBased Hemostatic Gauze Dressings--TCCC Guidelines-Change 13-05. J Spec Oper Med 2014;14:40-57.

10. Callaway DW, Smith ER, Cain J, et al. Tactical emergency casualty care (TECC): guidelines for the provision of prehospital trauma care in high threat environments. J Spec Oper Med 2011;11:104-22.

11. Kotwal RS, Montgomery HR, Kotwal BM, et al. Eliminating preventable death on the battlefield. Arch Surg 2011;146:1350-8.

12. Eastridge BJ, Mabry RL, Seguin P, et al. Death on the battlefield (2001-2011): implications for the future of combat casualty care. $J$ Trauma Acute Care Surg 2012;73:S431-7.

13. Pons P, Jacobs L. Save a life: What everyone should know to stop bleeding after an injury. Surg AC o(Ed) Google Sch 2016.

14. Bleeding Control. About Bleeding Control. http://www. bleedingcontrol.org/about-bc (Cited 18 Dec 2018).

15. Arksey H, O'Malley L. Scoping studies: towards a methodological framework. Int J Soc Res Methodol 2005;8:19-32.

16. Peters M, Godfrey C, Mclnerney P, et al. The Joanna Briggs Institute reviewers' manual 2015: methodology for JBI scoping reviews. 2015.

17. Tricco AC, Lillie E, Zarin W, et al. PRISMA Extension for Scoping Reviews (PRISMA-ScR): checklist and explanation. Ann Intern Med 2018;169:467-73.

18. Elsevier Solutions. Reference Manager and Academic Social Network - Mendeley Database. https://www.elsevier.com/solutions/ mendeley (Cited 18 Dec 2018).

19. Veritas Health Innovation. Covidence Systematic Review Software. Melbourne, Australia. 